\title{
PULMONARY STENOSIS WITH TRANSPOSITION OF THE GREAT VESSELS \\ BY
}

\author{
A. G. D. WHYTE AND J. McKENZIE
}

From the Department of Anatomy, University of Aberdeen

Congenital anomalies of the heart are now largely within the scope of treatment. A case history with detailed necropsy findings, especially when these are unusual and a developmental explanation is offered, may be worthy of record. This child survived five months with gross, apparently unusual, and largely unsuspected congenital cardiac lesions.

\section{CASE REPORT}

Born as a lusty class A boy on November 12, 1957, the child weighed $5 \mathrm{lb} .12 \mathrm{oz}$. at birth and showed no abnormality of any system during his seven days' stay in the Aberdeen Maternity Hospital. The mother's pregnancy had been normal except for a severe cold in the early months, and the labour, which was uncomplicated, lasted eight hours.

No significant disease was noted in the family history; the mother, aged 29 and mentally dull, suffered from chronic bronchitis; the father, aged 31, had a duodenal ulcer. The only other child, a poorly nourished boy aged 18 months, had had a cough since birth.

On February 19, 1958, the baby was admitted to the Royal Aberdeen Hospital for Sick Children for investigation of vomiting and cyanosis, but not as an emergency. The cyanosis, variable but aggravated by slight exertion, was becoming progressively worse. The child cried much and slept little, screaming when laid on his back, less cyanosed when propped up. A slow feeder, he often vomited soon after feeds. Attacks of "going stiff," with twitching of the limbs and marked cyanosis, were reported by the parents.

Although rather thin, the baby was about his expected weight. The cyanosis, noticeable when he was asleep, increased when he cried. A loud blowing systolic murmur, maximal in the fourth left interspace near the sternum, was transmitted round the chest wall to both bases. Rhonchi but no moist sounds could be heard. The systolic blood pressure reached $90 \mathrm{~mm}$. $\mathrm{Hg}$ and the femoral pulses could be felt. There was no clubbing of fingers or toes and the other systems were normal.

No increase of lung markings or cardiac enlargement could be seen on the radiograph, while screening failed to show any hypertrophy or overactivity but suggested pulmonary stenosis. The aorta was definitely left-sided.
Electrocardiography gave a high voltage tracing with right axis deviation and no evidence of bundle branch block.

With a provisional diagnosis of Fallot's tetralogy the baby was discharged from hospital on March 10, 1958. Admission was to be arranged later for further investigation including cardiac catheterization. However, at home the child developed an upper respiratory tract infection and died suddenly on April 24, 1958.

At necropsy, the thoracic contents were removed en bloc and the heart with its great vessels was examined in detail. The heart was enlarged, particularly the right atrium and the presumed right ventricle. The aorta, its normal branches arising from the arch, was displaced to the left with no sign of a pulmonary trunk on superficial inspection. Deep dissection between the aorta and the right atrium revealed a pulmonary vessel arising separately from the heart and dividing into two pulmonary arteries, the left passing under the aortic arch to reach the lung. A fibrosed ligamentum arteriosum was identified.

The right atrium was distended with firm blood clot, the superior and inferior venae cavae and coronary sinus opening normally into it. The foramen ovale was closed, but the atrial septum had a free arched lower border allowing free communication with a small left atrium receiving the pulmonary veins. The atrioventricular canal showed no sign of subdivision into right and left, but it possessed welldeveloped cusps. There was no ventricular septum and the walls of the common ventricular cavity were uniformly and markedly thickened (Fig. 1). The aortic opening and valve were normal although the coronary arteries were abnormally situated, the left arising from the left posterior sinus and the right from the right posterior sinus of the aorta. Immediately to the right of and behind the aorta, between it and the atrioventricular valve, lay the very narrow exit into the pulmonary circulation. Further dissection revealed that the boundaries of this opening were formed of closely approximated or tightly constricted muscular ridges. A few millimetres higher the pulmonary valve itself could be identified, severely stenosed, the cusps fused and projecting dome-like into the lumen of the vessel (Fig. 2).

Blood clot was found in the thoracic oesophagus but not in the stomach; no varices could be seen in the abdominal oesophagus and there was no other abnormality elsewhere. 


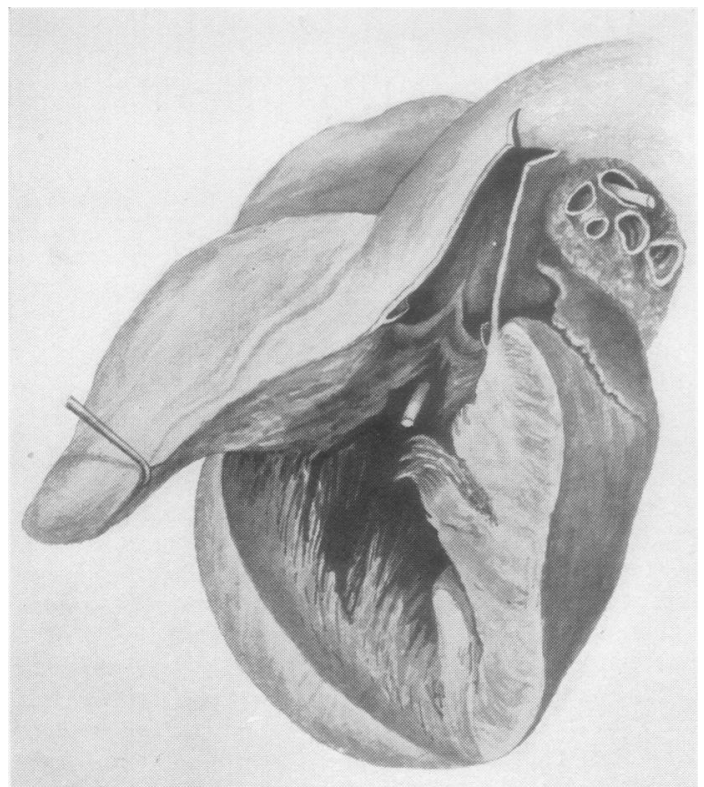

FIG. 1. The ventricular cavity showing marked thickening of the walls and the lack of any ventricular septum.

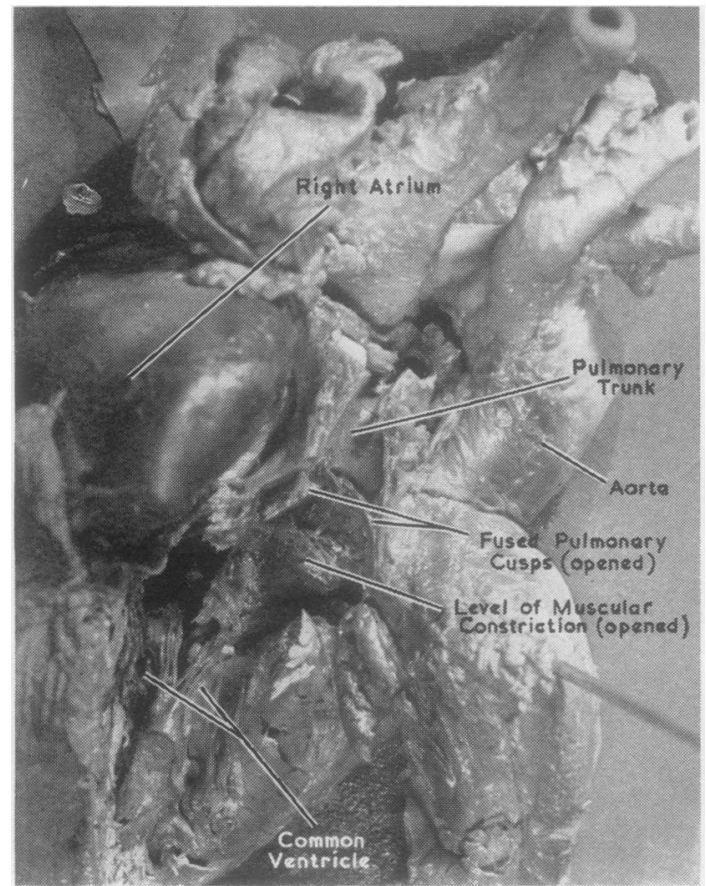

FIG. 2. The severely stenosed pulmonary valve with its fused cusps projecting into the lumen of the vessel.
The cardiac lesion then was multiple, consisting of transposition of the great vessels, absence of the ventricular septum, giving a common ventricle and a three-chambered heart, and pulmonary stenosis.

\section{Discussion}

We have been unable, in a review of the literature, to find a similar case showing this combination of abnormalities. In spite of the innumerable systems of classifying congenital heart disease, here is a group of defects which proved difficult to isolate in one compartment and may serve as a caution now that congenital anomalies of the heart are approached more with a view to remedy than with an eye on diagnosis only.

Of the congenital malformations of the heart, pulmonary stenosis with ventricular septal defect and hypertrophy of the right ventricle is a relatively common condition (Abbott, 1936 ; Wood, 1950). Transposition of the aorta and pulmonary artery does not occur so frequently, while ventricular septal defects vary in size and frequency, but the opening is seldom so large that a ventricular septum cannot be recognized; in fact Potter (1952) maintains that it "is never entirely absent in a heart whose ventricular development is otherwise normal." Nevertheless it was difficult, if not impossible, to decide which particular muscular ridge or buttress in this ventricle represented the septum.

Abbott (1936) notes that in complete transposition of the great trunks "where the ventricular septum is entire, the condition is of extreme gravity, only a small fraction of aerated blood reaching the systemic circulation and the unfortunate subjects dying in early infancy. An associated ventricular septal defect, on the other hand, provides distinctly improved circulatory conditions, in that a part of the venous blood from the right atrium passes directly through the defect into the transposed pulmonary artery and thence to the lungs for aeration; and a part of the oxygenated blood returned from the left atrium is transmitted an instant later through the same pathway into the transposed aorta and thence, mixed with unaerated blood from the right atrium, is distributed to the systemic circulation by the same shuttle-like action as is carried on to perfection in the heart of the turtle (i.e., two auricles and one common ventricle). Such patients may live into childhood or early adult life with fairly good cardiac efficiency and only moderate cyanosis. The larger the septal defect, the more 
easily can this circulatory arrangement be carried on. ...."

In this case the septal defect was complete, giving a common ventricle, but the associated pulmonary stenosis might have more than offset the advantage of the open septum cited by Abbott. Furthermore, stenosis of the pulmonary orifice was not compensated by patency of the ductus arteriosus and accordingly there was no easy way of filling the pulmonary system, but the wide pulmonary artery distal to the stenosis showed that some blood could and did escape into it from the ventricle. Whether this accounted for all the oxygenation that occurred or whether the bronchial arteries also helped significantly (Keith, 1909) it is impossible to say, but the mild cyanosis at rest showed that there was little or no reserve of oxygenated blood in the circulation. The marked deterioration in the physical condition of the child on admission to the Sick Children's Hospital compared with that on discharge from the Maternity Hospital several weeks before may be attributed to the closure of the ductus arteriosus in the interval.

In spite of the variety of defects in this specimen, it is instructive to realize that all of them stemmed from only one developmental disturbance in the formation of the heart, namely, in the subdivision of the truncus arteriosus by the truncus ridges (aortic septum). Appearing on opposite sides of the lumen of the truncus just distal to the origin of the pulmonary arteries (sixth aortic arches) these ridges spiral along the vessel towards the common ventricle. When they meet one another across the lumen the resultant subdivision of the vessel is also in spiral form, an aorta and a pulmonary trunk related to one another in the usual way. If these ridges were to take a straight course towards the heart, then the aortic portion would lie entirely in front of the pulmonary or just a little to one or other side of it, the condition found in this child.

These truncus ridges have still another part to play in the subdivision of the heart. In consort with contributions from the endocardial atrioventricular cushions and the connective tissue over the muscular ventricular septum, they normally close the gap between the ventricles. Since they provide the major contribution to the membranous septum, only the muscular portion remains to form a ventricular septum and even that may easily become submerged in muscular hypertrophy of the ventricle.

In his treatise on pulmonary stenosis, Brock (1957) does not refer to maldevelopment or malposition of the aortic septum as a cause of that defect, but the findings here do suggest that the development of the septum may play a part. In the first place, an abnormally developing septum has already been incriminated as the cause of the transposition, and it requires only a dorsal deflexion of the septum just above its junction with the developing aortico-pulmonary valves to reduce both the orifice of the pulmonary artery and the
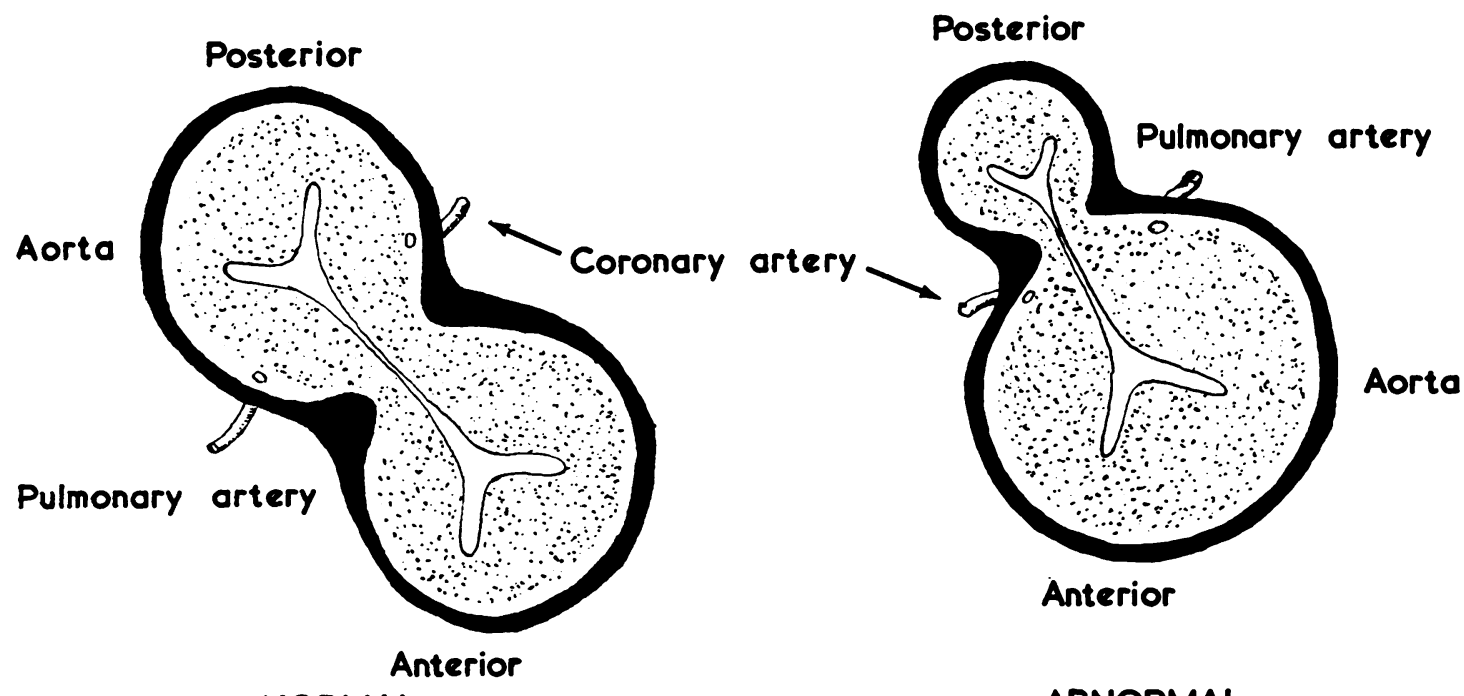

NORMAL

ABNORMAL

FIG. 3. Diagram illustrating maldevelopment of the aortic septum. 
size of the valve cusps. Secondly, also in support of this hypothesis, the two coronary arteries arose in this child from the sinuses of an abnormally situated aorta; with transposition of the great vessels, only a deflexion of the aortic septum towards the "pulmonary" side could allow this "normal" state of affairs (Fig. 3).

As Brock (1957) and others, e.g., Keith (1924), have shown, infundibular stenosis is frequently associated and often confused with pulmonary stenosis and, although the contribution of the bulbus cordis to the left ventricle is seldom in evidence, normally or abnormally, the muscular constriction below the pulmonary valve in this case closely resembled the anomaly as it occurs on the right side of the heart.

The pity is that this child was not fully investigated by modern methods. The results would have formed interesting and instructive correlation with the findings at necropsy.

Had there been the opportunity to operate, however, the surgical task may not have been too difficult or extensive ; the hazards of septal defect repair would not have been necessary in the light of Abbott's observations. Indeed, the aim in such a case would be the relatively efficient threechambered heart achieved by overcoming the "bulbar" and pulmonary stenosis.

We are indebted to Dr. D. M. Slessor for the opportunity to examine this child after death; to Professor Sir Dugald Baird, Aberdeen Maternity Hospital, and Professor John Craig, Royal Aberdeen Hospital for Sick Children, for permission to consult the records in these hospitals, and to Professor R. D. Lockhart for his help and encouragement in the preparation of the paper.

\section{REFERENCES}

Abbott, M. E. (1936). Atlas of Congenital Cardiac Disease. American Heart Association, New York.

Brock, R. C. (1957). The Anatomy of Congenital Pulmonary Stenosis. Cassell, London.

Keith, A. (1909). Lance+, 2, 359, 433, 519.

- (1924). Ibid., 2, 1267.

Potter, E. L. (1952). Pathology of the Fetus and the Newborn. Year Book Publishers, Chicago.

Wood, P. (1950). Brit. med. J., 2, 639, 693. 